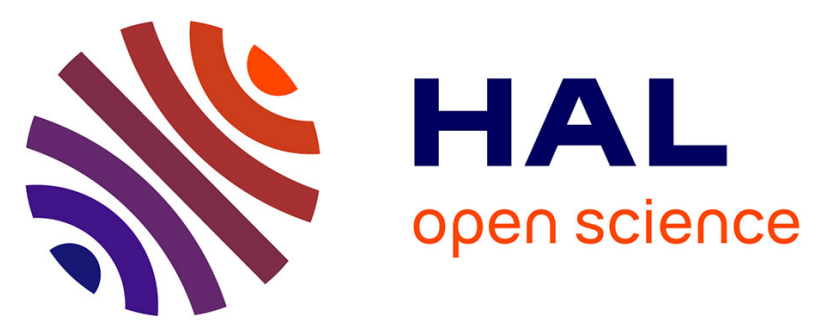

\title{
Non Intrusive Global/Local Coupling Techniques in Solid Mechanics: An Introduction to Different Coupling Strategies and Acceleration Techniques
}

Olivier Allix, Pierre Gosselet

\section{- To cite this version:}

Olivier Allix, Pierre Gosselet. Non Intrusive Global/Local Coupling Techniques in Solid Mechanics: An Introduction to Different Coupling Strategies and Acceleration Techniques. L. De Lorenzis and A. Düster. Modeling in Engineering Using Innovative Numerical Methods for Solids and Fluids, 599, Springer Nature Switzerland AG, pp.203-220, 2020, CISM International Centre for Mechanical Sciences - Courses and Lectures, 978-3-030-37517-1. 10.1007/978-3-030-37518-8_6 . hal-02476964

\author{
HAL Id: hal-02476964 \\ https://hal.science/hal-02476964
}

Submitted on 13 Feb 2020

HAL is a multi-disciplinary open access archive for the deposit and dissemination of scientific research documents, whether they are published or not. The documents may come from teaching and research institutions in France or abroad, or from public or private research centers.
L'archive ouverte pluridisciplinaire HAL, est destinée au dépôt et à la diffusion de documents scientifiques de niveau recherche, publiés ou non, émanant des établissements d'enseignement et de recherche français ou étrangers, des laboratoires publics ou privés. 


\title{
Non intrusive Global/local coupling techniques in solid mechanics: an introduction to different coupling strategies and acceleration techniques
}

\author{
Olivier Allix ${ }^{*}$ and Pierre Gosselet ${ }^{\dagger}$ \\ ${ }^{*}$ LMT, ENS Paris-Saclay/CNRS \\ ${ }^{\dagger}$ LaMcube, Univ. Lille/CNRS/Centrale Lille
}

February 13, 2020

\begin{abstract}
The aim of this paper is to provide, for a reader not familiar with the non-intrusive coupling method, the simplest possible example on which all the different iterative coupling strategies can be solved by hands. Among them, the basic algorithm, Aitken's method, mixed interface conditions... A drawback of this example is that, for some acceleration techniques, the convergence can be achieved in one iteration after the initialization. Nevertheless it allows to easily become acquainted with the different techniques. Some examples from previous papers are then used to illustrate the same properties on more complex examples involving nonlinearity.
\end{abstract}

\section{Introduction}

In the last decade, many innovative modeling or solution techniques have been introduced in the field of computational mechanics. These techniques, such as enriched finite elements or multiscale modeling, enable to perform complex simulations that are out of the reach of conventional finite element analysis (FEA) tools, in terms of computational or human costs. Although these techniques have proved their performance by extensive testing on academic applications, they are scarcely applied on actual industrial problems because they cannot be conveniently implemented into commercial FEA software packages. Therefore, a scientific and practical challenge is to allow realistic simulation of complex industrial problems including all their physical and technological complexity. A view on this issue can be found in a prospective document of the NSF blue-ribbon panel Oden et al. (2006):

"If an industry is to replace testing with simulation, the simulation tools must undergo robust verification and validation procedures for effectiveness. Overall, simulation in industry has yet to meet its full potential. The following list is a summary of its current limitations:

1. The development of models is very time-consuming, particularly for geometries of complex engineering systems $[\ldots]$

2. Methods are needed for linking models at various scales and simulating multi-physics phenomena."

We are interested in the case where the complex phenomenon to be analyzed concerns one or several reduced parts (called local models) of the whole body (call global model). Those parts often correspond to very fine structural details that can not be taken into account in the global mesh of the structure, details which in turns can induce nonlinearity such as plasticity, visco-plasticity or damage. A typical example is presented in Figure 1 where one can see that a proper description of the local parts may require meshes larger than the mesh used for the global model Blanchard et al. (2019).

A method often used by engineers to tackle such problems is the submodeling approach Cormier et al. (1999): after the global computation, structural zooms are applied on the local critical zones, with details represented exactly. An advantage of this approach is that it allows to easily connect research software and commercial code, as was done for example to deal with the prediction of delamination under low velocity impacts Allix (2001). Unfortunately, it implies neglecting the influence of the local zones on the whole structure. This may lead in turn to quite important local errors. The problem becomes more crucial when nonlinearity initiated locally spreads over the whole structure. 


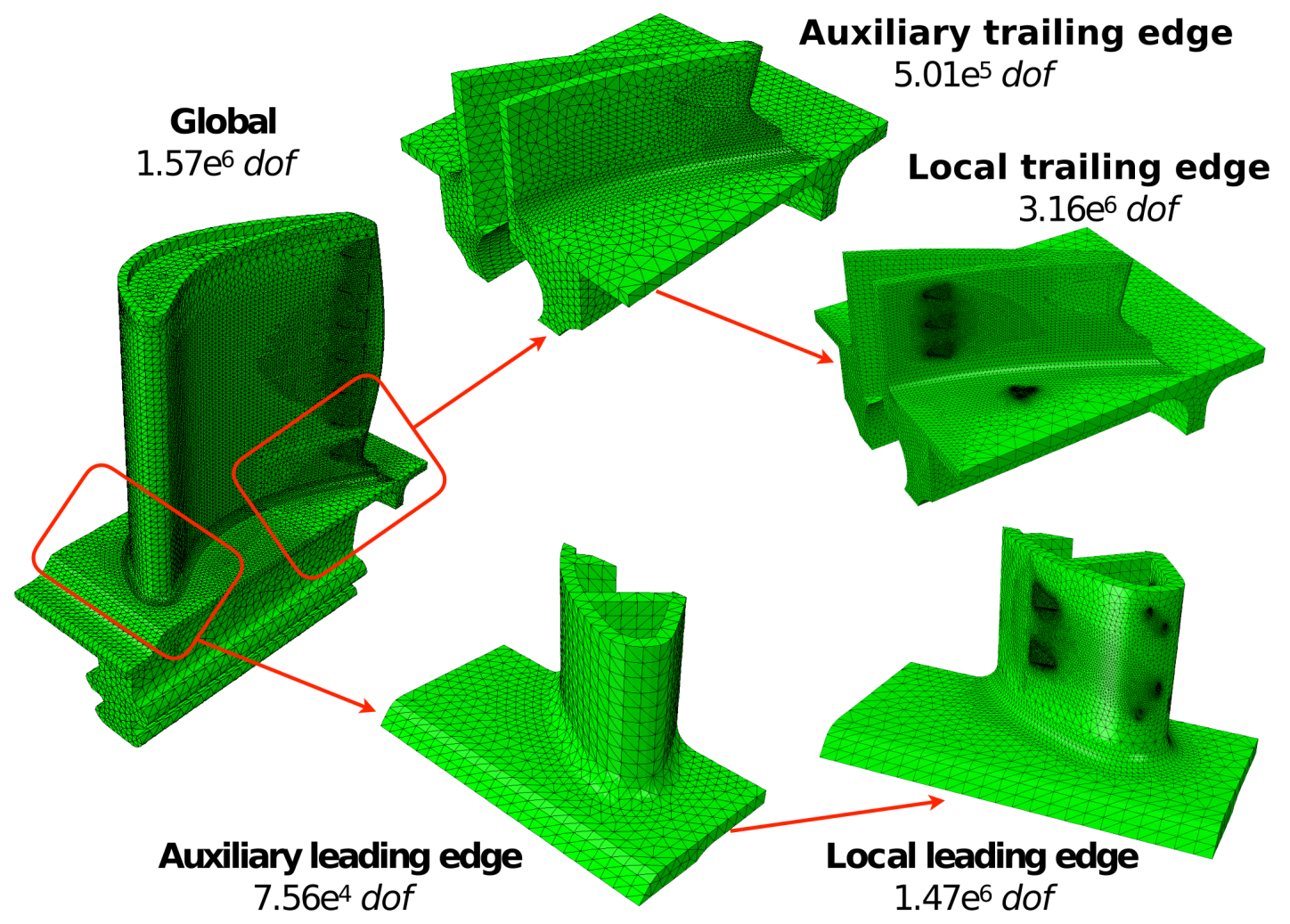

Figure 1: Illustration of global and locals models.

To correct the drawback of submodeling while keeping its simplicity and flexibility, a non-invasive method was proposed in order to allow exact local/global analysis, embedding the same basic tools as those used in the submodeling inside an iterative procedure. The prerequisite of the proposed framework is to keep unchanged the global numerical model as well as the solver used for its treatment. Therefore, two or several models are used concurrently, the untouched global model and the local ones which are iteratively substituted where needed. The exchanges between the models are such that the data should be "natural" to the software, such as prescribed nodal reactions or displacements.

The proposed method aims therefore at converging by iterations toward the reference problem by means of submodeling-like steps. The formulation of the method and its numerical optimization have first been derived in the case of global linear models and local plasticity Gendre et al. (2009, 2011). A number of other applications and extensions have been proposed: use of XFEM at the local scale Passieux et al. (2013), treatment of non-matching interfaces Liu et al. (2014), coupling between a global plate model and 3D parts for bolted assemblies Guguin et al. (2014), geometrically non conforming coupling Guinard et al. (2018), multiscale time and space computation in explicit dynamic Bettinotti et al. (2014) with implementation in Abaqus Explicit for the analysis of delamination under impact Bettinotti et al. (2017), non-invasive domain decomposition approach Duval et al. (2016). Mesh refinement based on error estimation may also be cast in the proposed non-intrusive framework Duval et al. (2018).

Alternative proposals exist, based on volume coupling as for example the Arlequin method Dhia (1998). The implementation of such methods in a legacy code is not straightforward, mainly because the creation of the coupling operators between the two models in the transition zone requires complex integration operations. The volume coupling may also be performed by means of a non-invasive version of the Partition of Unity method Plews et al. (2012); Fillmore and Duarte (2018), by using projection techniques between the local and global models Temizer and Wriggers (2011); Holl et al. (2013) or by means of homogenization-like techniques Hühne et al. (2016).

The aim of this paper is to provide, for a reader not familiar with the non-intrusive coupling method, the simplest possible example on which most of the different iterative coupling strategies used in the previously cited papers can be solved by hands. Among them, the basic algorithm, Aitken's method, mixed interface conditions... A drawback of this example is that, for some acceleration techniques, the convergence is achieved in one iteration after the initialization. Nevertheless, it allows to easily become 
acquainted with the different techniques. For this we consider the case of a bar in tension as described on Figure 2.

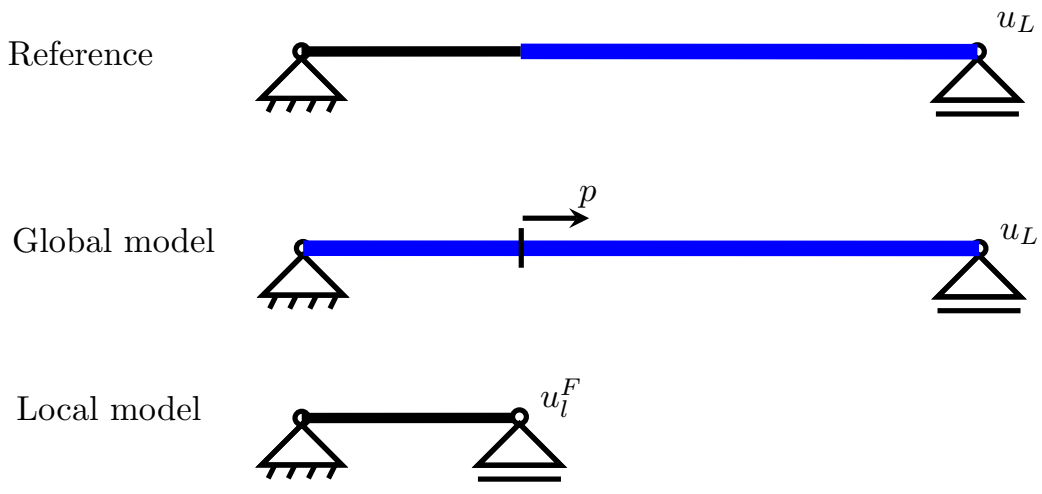

Figure 2: Reference, Global and Local models

\section{Reference model}

Let us consider a unit-section beam, clamped on its left side $(x=0)$ and with imposed traction displacement $u_{L}$ on its right side $(x=L)$. The beam is made out of two components: the Young modulus of the left part $[0, l]$ is $E_{F}$, while it is $E_{G}$ on the right side $[l, L]$. We use the ' notation for the derivative with respect to the axial coordinate $\mathrm{x}$. The subscript $R$ is used for the reference solution. In what follows we separate the left $(-)$ and right $(+)$ sides of the interface point $x=l$. The system of equations satisfied by the Reference displacement $u^{R}$ and the Reference tension $\sigma^{R}$, can be written as:

$$
\begin{array}{rlrl}
\sigma^{R} & \left.=E^{F}\left(u^{R}\right)^{\prime} \text { in }\right] 0, l[ & \sigma^{R} & \left.=E^{G}\left(u^{R}\right)^{\prime} \text { in }\right] l, L[ \\
\sigma^{R^{\prime}} & =0 \text { in }] 0, l[\text { and in }] l, L[ & & \\
u^{R}(0) & =0 & & \\
u_{-}^{R}(l) & =u_{+}^{R}(l) & & =u_{L} \\
u_{-}^{R}(l) & =\sigma_{+}^{R}(l)
\end{array}
$$

The solution is uniform in tension, and continuous piecewise-linear in displacement:

$$
\begin{aligned}
u^{R}(x) & =u_{l}^{R} \frac{x}{l} \text { in }[0, l] \\
u^{R}(x) & =u_{l}^{R}+\left(u_{L}-u_{l}^{R}\right) \frac{x-l}{L-l} \text { in }[l, L] \\
u_{l}^{R} & =u_{L} \frac{1}{1+\frac{E^{F}}{E^{G}} \frac{L-l}{l}} \\
\sigma^{R} & =\frac{E^{F} u_{l}^{R}}{l}=\frac{E^{F} E^{G}}{E^{G} l+E^{F}(L-l)} u_{L}
\end{aligned}
$$

\section{Iterative techniques using the global and the local models separately}

As previously described, the principle of the method is to use the two models described on Figure 2. The local model, to which a displacement coming from a previous global solution is imposed at the interface, and the global one where an extra load is prescribed at the interface. The different iterative techniques aim basically at determining the load $p$ to be prescribed to the global model which would lead to the exact solution, in the unchanged part of the global model (referred to as complement zone) and in the local model. To avoid possible misunderstanding we first define the two models. Let us also note that the convergence properties presented on this simple example can be generalized to structural problems introducing the Schur complement of the different domains Gosselet et al. (2018a). 


\subsection{Local model}

The Local model is the extraction of the left part of the Reference model. In order to avoid confusion between the point $L$ and the Local model, we use the F superscript for the Local model, meaning Fine model. A Dirichlet condition $u_{l}^{F}$ is imposed on the right side $x=l$ of the Fine model:

$$
\begin{aligned}
\sigma^{F} & \left.=E^{F} u^{\prime} \text { in }\right] 0, l[ & & \left.\left(\sigma^{F}\right)^{\prime}=0 \text { in }\right] 0, l[ \\
u^{F}(0) & =0 & & u^{F}(l)=u_{l}^{F}
\end{aligned}
$$

and the solution is:

$$
\begin{aligned}
u^{F}(x) & =u_{l}^{F} \frac{x}{l} \text { in }[0, l] \\
\sigma^{F} & =\frac{E^{F} u_{l}^{F}}{l} \text { in }[0, l]
\end{aligned}
$$

\subsection{Global model}

The Global model is a simplification of the Reference model with a coarse representation of the zone of interest $[0, l]$. In our case, we chose a homogeneous beam with Young modulus $E^{G}$. An extra effort $p^{G}$ is imposed at the interface $x=l$ :

$$
\begin{aligned}
\sigma^{G} & \left.=E^{G}\left(u^{G}\right)^{\prime} \text { in }\right] 0, L[ & & \\
\left(\sigma^{G}\right)^{\prime} & =0 \text { in }] 0, l[\text { and in }] l, L[ & & \\
u^{G}(0) & =0 & u^{G}(L) & =u_{L} \\
u_{-}^{G}(l) & =u_{+}^{G}(l) & \sigma_{-}^{G}(l) & =\sigma_{+}^{G}(l)+p^{G}
\end{aligned}
$$

The solution can be written as:

$$
\begin{aligned}
u^{G}(x) & =u_{l}^{G} \frac{x}{l} \text { in }[0, l] \\
u^{G}(x) & =u_{l}^{G}+\left(u_{L}-u_{l}^{G}\right) \frac{x-l}{L-l} \text { in }[l, L] \\
u_{l}^{G} & =\left(\frac{p^{G}(L-l)}{E^{G}}+u_{L}\right) \frac{l}{L} \\
\sigma_{+}^{G} & =\frac{E^{G} u_{L}}{L}-p^{G} \frac{l}{L}
\end{aligned}
$$

The coarse representation of the zone of interest in the Global model $[0, l]$ was often called Auxiliary model in previous papers, here it is noted with subscript -. The zone $[l, L]$ where the Global and Reference models match is the Complement zone, here written with subscript + .

Remark 1 (Solution in term of $\left.p^{G}\right)$. By comparing (6) and (2), we see that $\sigma_{+}^{G}=\sigma^{R}$ can be achieved for a specific value of $p^{G}$, named $p^{R}$ :

$$
\begin{gathered}
\sigma^{R}=\frac{E^{F} E^{G}}{E^{G} l+E^{F}(L-l)} u_{L}=\frac{E^{G} u_{L}}{L}-p^{R} \frac{l}{L}=\sigma_{+}^{G} \\
p^{R}=\frac{\left(E^{G}-E^{F}\right)}{E^{G} l+E^{F}(L-l)} E^{G} u_{L}
\end{gathered}
$$

where we see that, of course, $p^{R}$ is proportional to the dissemblance between the Fine and the Coarse model in the zone of interest.

\subsection{Basic fixed point iterative technique}

The basic iteration consists in a Global computation for a given $p_{i}^{G}$, from which we deduce the displacement to be imposed on the Local model. We then evaluate the lack of balance between the Local model and the Complement zone of the Global model, this residual, written $r$, is to be added to $p_{i}^{G}$ to define the next load $p_{i+1}^{G}$ of the Global model. 


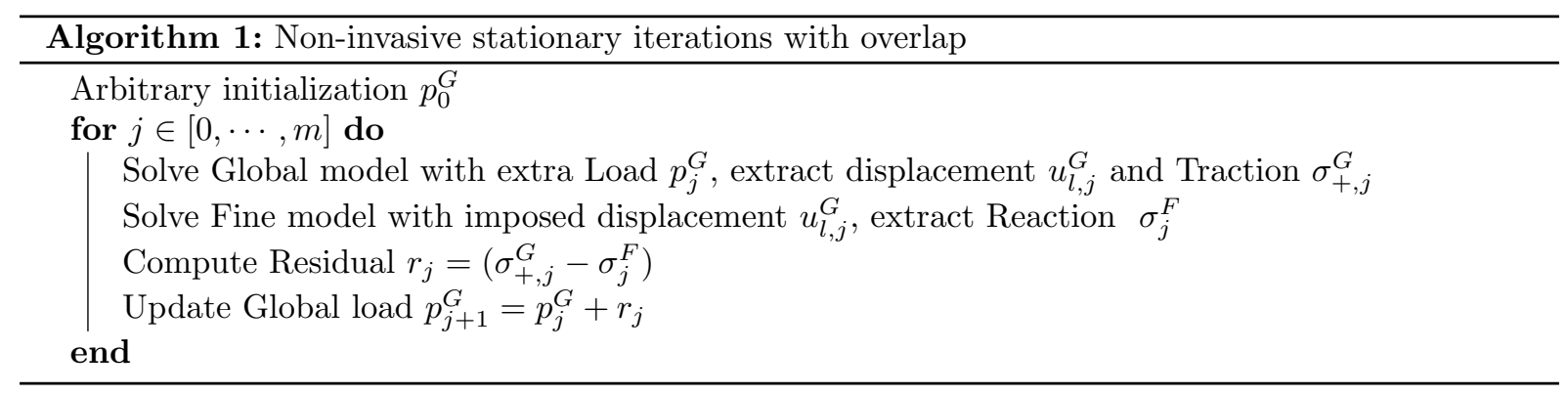

Using previous formula, we have:

$$
\begin{aligned}
p_{j+1}^{G} & =p_{j}^{G}+r_{j}=p_{j}^{G}+\left(\sigma_{+, j}^{G}-\sigma_{j}^{F}\right) \\
& =p_{j}^{G}-\frac{E^{F} u_{l, j}^{G}}{l}-p_{j}^{G} \frac{l}{L}+\frac{E^{G} u_{L}}{L} \\
& =p_{j}^{G}-\frac{E^{F}\left(\frac{p_{j}^{G}(L-l)}{E^{G}}+u_{L}\right) \frac{l}{L}}{l}-p_{j}^{G} \frac{l}{L}+\frac{E^{G} u_{L}}{L} \\
& =p_{j}^{G}\left(1-\frac{E^{F}}{E^{G}}\right) \frac{L-l}{L}+u_{L}\left(\frac{E^{G}-E^{F}}{L}\right)
\end{aligned}
$$

We recognise a fixed point iteration. We remind below the classical conditions of convergence of a fixed point algorithm; we will then make used of similar results for the other algorithms presented in this paper.

Proposition 2 (Condition of convergence). The iteration is a contraction and it converges if

$$
\rho=\left|\left(1-\frac{E^{F}}{E^{G}}\right) \frac{L-l}{L}\right|<1
$$

In fact:

$$
\begin{aligned}
& u_{L}\left(\frac{E^{G}-E^{F}}{L}\right)=p_{j+1}^{G}-p_{j}^{G}\left(1-\frac{E^{F}}{E^{G}}\right) \frac{L-l}{L} \\
& u_{L}\left(\frac{E^{G}-E^{F}}{L}\right)=p_{j}^{G}-p_{j-1}^{G}\left(1-\frac{E^{F}}{E^{G}}\right) \frac{L-l}{L}
\end{aligned}
$$

Thus by subtracting the previous two relations we obtain, whatever $j \geq 1$ :

$$
p_{j+1}^{G}-p_{j}^{G}=\left(1-\frac{E^{F}}{E^{G}}\right) \frac{L-l}{L}\left(p_{j}^{G}-p_{j-1}^{G}\right)
$$

Thus:

$$
p_{j+1}^{G}-p_{j}^{G}=\left(\left(1-\frac{E^{F}}{E^{G}}\right) \frac{L-l}{L}\right)^{j}\left(p_{1}^{G}-p_{0}^{G}\right)
$$

Thus, if $\rho<1$, then $\left(p_{j}^{G}\right)$ is a Cauchy sequence. As we work in a complete space, $\left(p_{j}^{G}\right)$ tends to the limit $p_{\infty}^{G}$. The convergence is linear with rate $\rho$.

Proposition 3 (Limit). If the iteration is a contraction, we recover (7) when searching $p_{\infty}^{G}=p^{R}$.

In fact, from the previous relation $p_{\infty}^{G}$ satisfies:

$$
\begin{array}{r}
p_{\infty}^{G}=p_{\infty}^{G}\left(1-\frac{E^{F}}{E^{G}}\right) \frac{L-l}{L}+u_{L}\left(\frac{E^{G}-E^{F}}{L}\right) \\
p_{\infty}^{G}=\frac{\left(E^{G}-E^{F}\right)}{E^{G} l+E^{F}(L-l)} E^{G} u_{L}=p^{R}
\end{array}
$$

Remark 4. The case where the basic fixed point iteration diverges corresponds to two cases (the case $l=0$ is excluded):

$$
\begin{gathered}
\left(1-\frac{E^{F}}{E^{G}}\right) \frac{L-l}{L}>1 \Leftrightarrow-\frac{l}{L-l}>\frac{E^{F}}{E^{G}} \\
\left(1-\frac{E^{F}}{E^{G}}\right) \frac{L-l}{L}<-1 \Leftrightarrow 1+\frac{L}{L-l}<\frac{E^{F}}{E^{G}}
\end{gathered}
$$


In those cases, it is required to make use of more refined algorithms. The first case corresponds practically to the possibility of softening. This is why relaxation was used in Gerasimov et al. (2018) where a local model prone to cracking was modeled by means of a phase field approach of fracture. The second case corresponds to a local material much stiffer than the global one.

\subsection{Basic fixed point with relaxation}

In order to improve the convergence rate, one can simply use relaxation. For a given $\omega \in \mathbb{R}^{+}$, the update formula is modified:

$$
p_{j+1}^{G}=p_{j}^{G}+\omega r_{j}
$$

and the rate of convergence can be computed, and minimized in order to obtain the optimal relaxation:

$$
\begin{aligned}
\rho & =1-\omega \frac{E^{G} l+E^{F}(L-l)}{E^{G} L} \\
\omega_{o p t} & =\frac{E^{G} L}{E^{F}(L-l)+E^{G} l}
\end{aligned}
$$

\subsubsection{Aitken's acceleration}

Aitken's acceleration can be viewed as an automatic tuning of the relaxation parameter using the formula:

$$
\omega_{j+1}^{\text {aitken }}=-\omega_{j}^{\text {aitken }} \frac{r_{j-1}\left(r_{j}-r_{j-1}\right)}{\left\|r_{j}-r_{j-1}\right\|}
$$

where an initial relaxation must be provided (in general equal to 1).

In this very simple case (scalar unknown and linear problem), it can be checked, using equations 6 and 8, that Aitken's formula reaches the optimal relaxation after the first iteration.

\subsubsection{Robin condition on the Fine model}

A possibility, in order to obtain fast convergence, is to improve the boundary condition applied to the Fine model, see Figure 3. Indeed, a Dirichlet condition has the advantage of being easy to implement, and always available in legacy codes, but it provides an extremely simplified vision of the Complement domain.

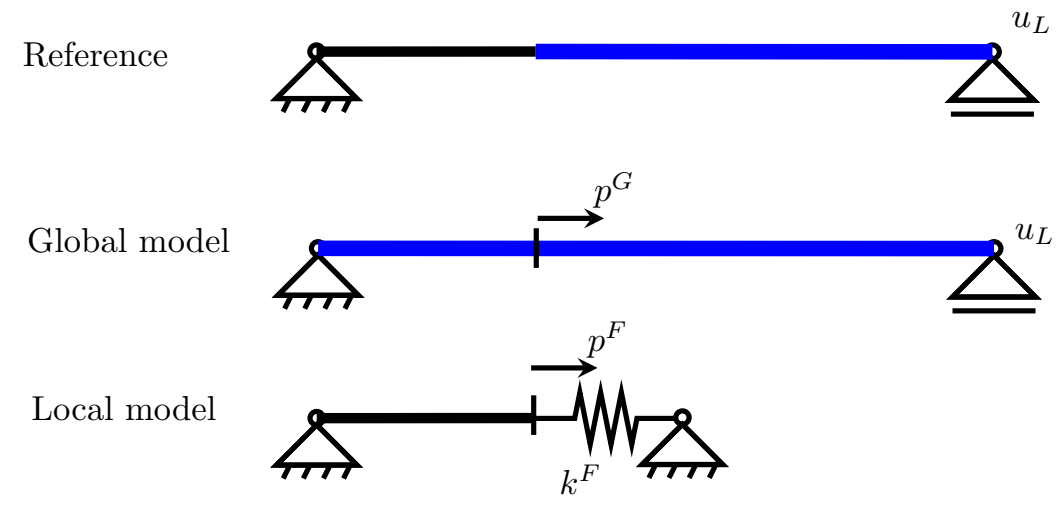

Figure 3: Reference, Global and Local models

In this approach, not only reactions are not balanced $\left(\sigma_{+}^{G}-\sigma^{F}\right) \neq 0$, but the local displacement $u^{F}(l)$ does not match the global displacement $u^{G}(l)$, unless convergence is reached. We gather the two conditions in an equivalent form:

$$
\begin{aligned}
& \left(\sigma_{+}^{G}-\sigma^{F}\right)-k^{F}\left(u^{F}(l)-u^{G}(l)\right)=0 \\
& \left(\sigma_{+}^{G}-\sigma^{F}\right)-k^{S}\left(u^{G}(l)-u^{F}(l)\right)=0
\end{aligned}
$$

where $k^{F}$ and $k^{S}$ are parameters homogeneous to a stiffness. The first expression is used to define the boundary condition on the local model whereas the second is used to evaluate the residual which should take into account not only the lack of balance of forces but also the jump of displacements. 
We consider the modified Fine model:

$$
\begin{aligned}
& \left.\sigma^{F}=E^{F}\left(u^{F}\right)^{\prime} \text { in }\right] 0, l\left[\quad\left(\sigma^{F}\right)^{\prime}=0 \text { in }\right] 0, l[ \\
& u^{F}(0)=0 \quad \sigma^{F}(l)+k^{F} u^{F}(l)=k^{F} u^{G}(l)+\sigma_{+}^{G}
\end{aligned}
$$

If we note $p^{F}=k^{F} u^{G}(l)+\sigma_{+}^{G}$, we recover the configuration of Figure 3. The solution is:

$$
\begin{aligned}
u^{F}(x) & =\frac{p^{F}}{k^{F} l+E^{F}} x \text { in }[0, l] \\
\sigma^{F} & =\frac{p^{F} E^{F}}{k^{F} l+E^{F}} \text { in }[0, l]
\end{aligned}
$$

As said earlier, the second line of (18) is used to define the a residual which measures both the lack of balance and of continuity between the Local and Global models at the interface:

$$
r_{j}=\left(\sigma_{+, j}^{G}-\sigma_{j}^{F}\right)+k^{S}\left(u_{l, j}^{F}-u_{l, j}^{G}\right)
$$

This leads to Algorithm 2. If we analyze one iteration, we get:

$$
\begin{aligned}
p_{j+1}^{G} & =p_{j}^{G}+r_{j} \\
& =p_{j}^{G} \frac{l\left(E^{G}-E^{F}\right)\left((L-l) k^{F}-l k^{S}\right)-E^{F} L\left(k^{S} l-E^{G}\right)}{\left(k^{F} l+E^{F}\right) E^{G} L} \\
& \ldots+\frac{\left(E^{G}-E^{F}\right)\left(k^{F}+k^{S}\right) l u_{L}}{\left(k^{F} l+E^{F}\right) L}
\end{aligned}
$$

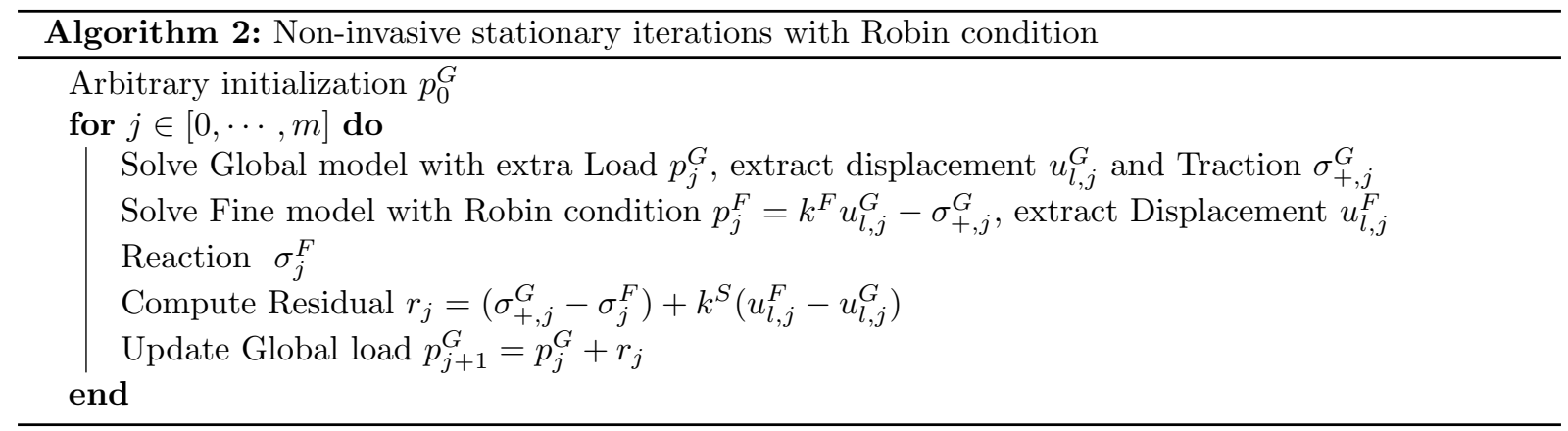

The optimal parameters are $k^{F}=\frac{E^{G}}{L-l}$, that is to say the equivalent stiffness of the complement zone, and $k_{S}=\frac{E^{G}}{l}$ the stiffness of the Global representation of the zone of interest. With these parameters, convergence is obtained in one iteration. Indeed, choosing these values one obtains:

$$
p_{j+1}^{G}=0 * p_{j}^{G}+p^{R}
$$

In fact there exists a whole range of admissible mixed parameters ensuring the convergence, which can be fully characterized using Proposition 2.

Remark 5. It can be shown that all the properties of different versions of the coupling only depends on the equivalent stiffness of the domains: Fine model $S^{F}=E^{F} / l$, Complement domain $S^{C}=E^{G} /(L-l)$, Auxiliary model (coarse representation of the zone of interest in the Global model) $S^{A}=E^{A} / l$. More, the equivalent stiffness of a beam is generalized by the concept of Schur complement, and with minor caution, all the results above can be generalized to $2 D$ ou $3 D$ elasticity. Note that in practice using the optimal $k_{S}$ is not a problem whereas estimating the optimal $k^{F}$ is much more involved. The question of finding a good Robin condition mimicking the stiffness of a given domain has been addressed in many papers. In the frame of non-intrusive coupling, a two-scale approximation was proposed and tested in Gendre et al. (2011).

\subsection{Use of overlap}

This version of the method was proposed to handle certain incompatibilities of the models: use of nonmatching meshes Gosselet et al. (2018b) or models with different dimensionality (Global plate vs Fine 3D) Guguin et al. (2014). 


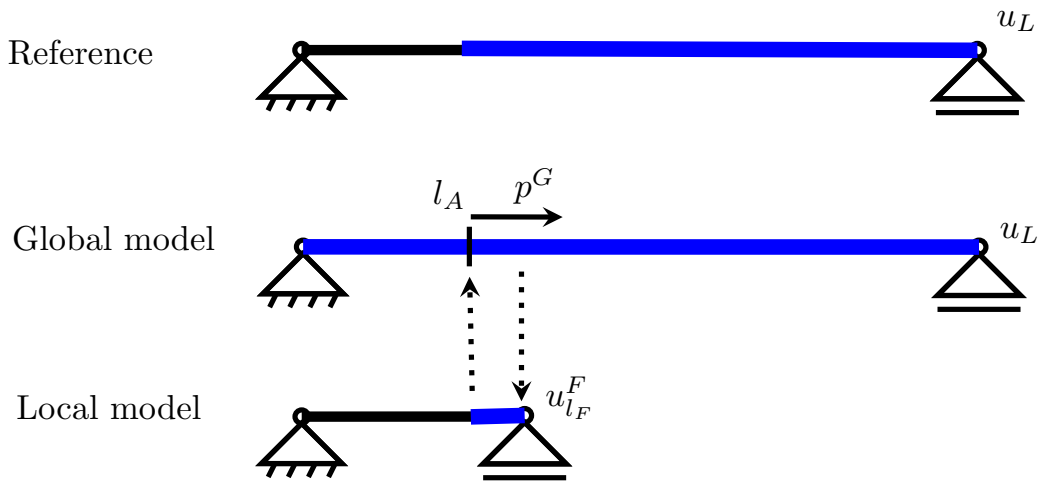

Figure 4: Reference, Global and Local models with overlap

We distinguish two inner boundaries: let $l_{F}$ be the limit of the Fine model and $l_{A}<l_{F}$ be such that the Global model in $\left[l_{A}, L\right]$ matches the Reference model. The extra traction on the Global model is imposed at position $l_{A}$ whereas the displacement to be imposed on the Fine model is obtained at position $l_{F}$. See figure 4 .

Note that if the models do not match exactly in the overlap, then the limit of the iterations might differ from the Reference model. In the case where the Reference is well characterized this might be a problem. In many circumstances (non matching meshes, models of different dimensionality) there is no real reference and the limit of the iterations gives a mechanically sound coupled model.

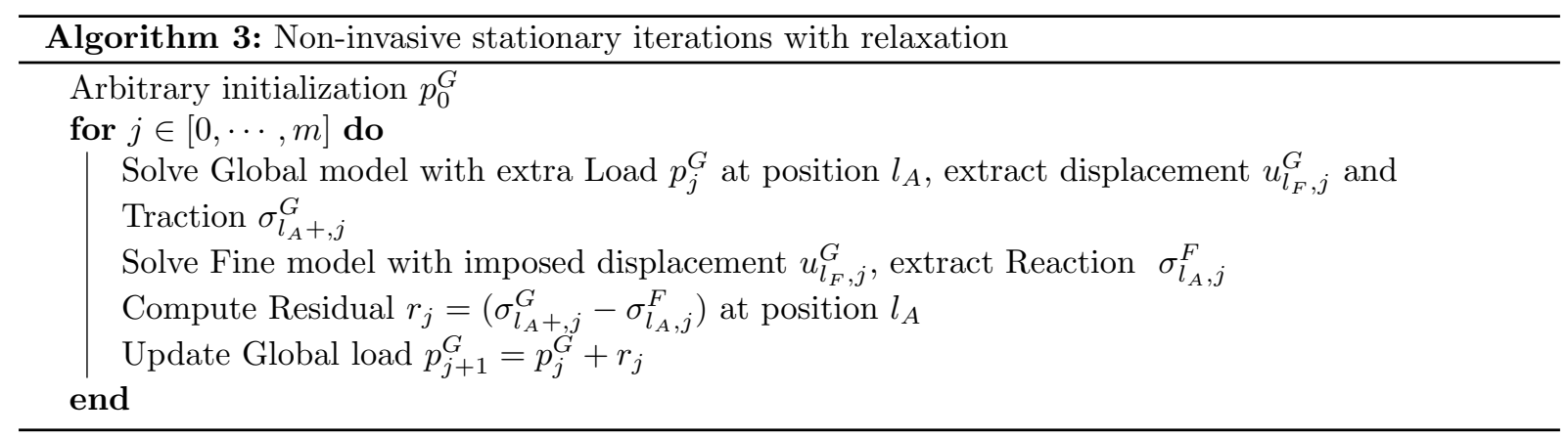

\section{Illustrations}

\subsection{Application of the previous results}

We illustrate previous study for the following values: $L=1, u_{L}=1 / 10, l=L / 4, E^{G}=1, E^{F}=.75$.

In Figure 5, we compare the relaxation techniques. Note that in this simple case with 1D interface, optimal relaxation leads to convergence in on iteration, Aitken's formula finds the optimal relaxation as soon as it possibly can (iteration 2).

In Figure 6, we compare the convergence for different values of the Robin parameters. The basic (primal) iteration is printed, it corresponds to $k^{S}=0$ and $k^{F}=\infty$. The optimal setting leads to convergence at the first iteration. It appears that the convergence rate is more sensitive to variations in $k^{S}$ than in $k^{F}$, which is lucky since the computation of the optimal $k^{S}$ is actually feasible. As soon as $k^{S}$ is well-chosen, a wide range of values of $k^{F}$ leads to better convergence than basic iteration.

In Figure 7, we compare the convergence for different lengths of the overlap. Large overlap is needed to ensure significant speedup. Using overlap is thus not a competitive acceleration technique. Its interest mostly lies in its capability to handle non-conforming meshes or models.

\subsection{Comments on other iterative algorithms}

This subsection briefly presents other iterations that have been tested but can not be illustrated on the simple 1D linear example. 


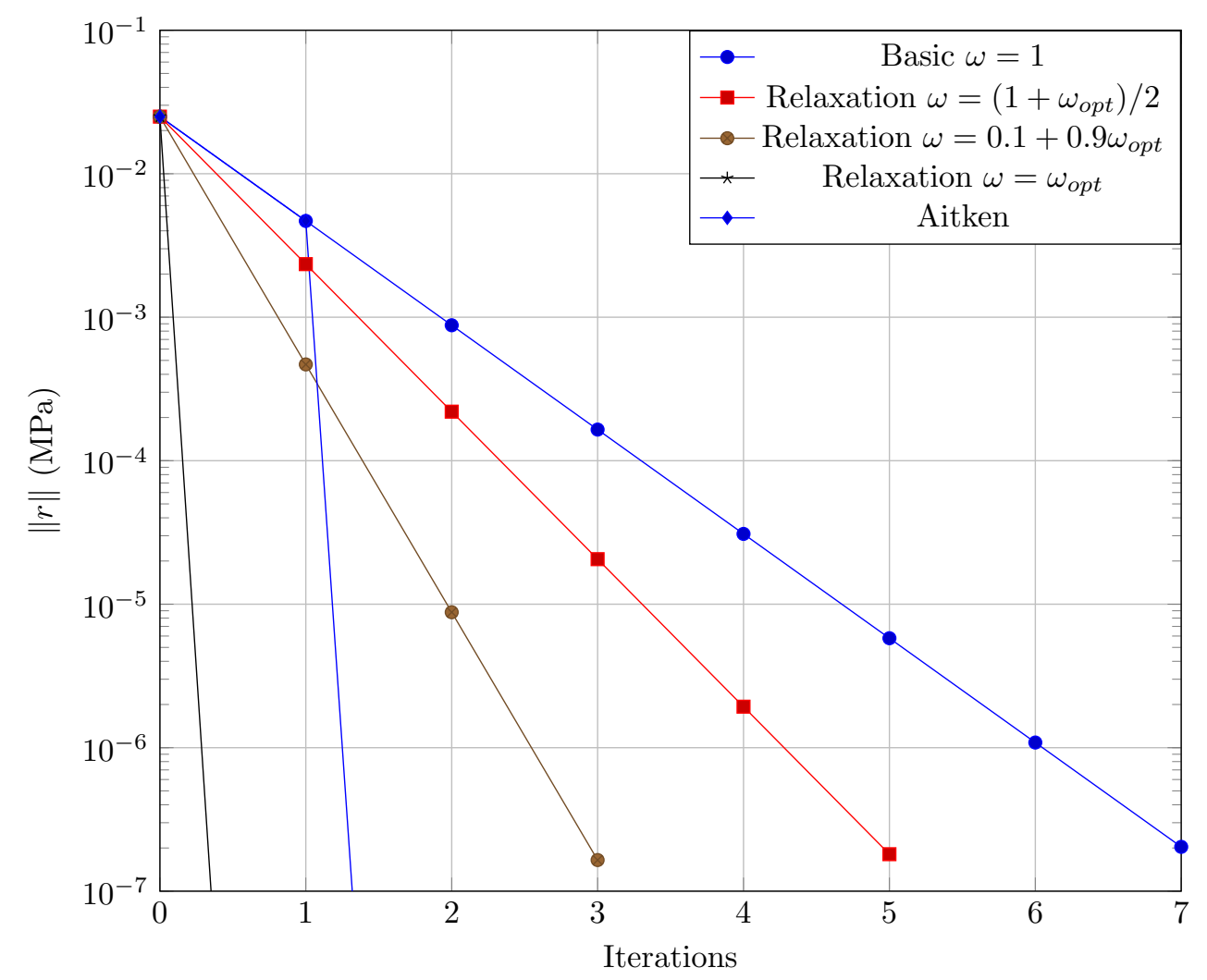

Figure 5: Comparison between relaxation techniques

In the linear case, the fixed point can be accelerated by a Krylov solver. The particular structure of the fixed point operator, which can be written as the matrix of the reference problem preconditioned by the global problem, makes it possible to apply a conjugate gradient algorithm Gosselet et al. (2018b). Since the Global problem often forms an excellent preconditioner for the reference problem, the convergence is extremely fast.

In the nonlinear case, if the Global model remains linear, quasi-Newton techniques like BFGS or SR1 can naturally be applied Gendre et al. (2009). Again, because of the quality of the Global model, linesearch appears not to be mandatory which enabled us to propose a fully nonlinear version of quasi-Newton Gosselet et al. (2018b). Also, nonlinear conjugate gradient was tested with interesting performance if carefully configured Gosselet et al. (2018b).

Anyhow, in almost all the cases we ever tested, it appeared that Aitken's $\Delta^{2}$ formula provided excellent performance in term of wall clock time, for an extremely simple implementation.

In the case of studies defined over large (pseudo)time intervals where the computation must be carried out on a succession of time steps. The use of partially decoupled time scales between the models was studied in Blanchard et al. (2019).

\section{$53 \mathrm{D}$ example}

In order to illustrate the method on a more significant test case, we propose to use the data from Gendre et al. (2011). The structure is the "sweded" turbine blade presented in Figure 8, it was provided by Safran Aircraft Engines and it is representative of actual engineering work. In this particular case, only the mechanical behavior of the zone of interest is altered in the Global model: the Fine model is elastoplastic whereas the Global model is purely elastic. Meshes are unchanged in the different models. Again, the constitutive relations are representative of actual problems. In general industrialists address this kind of problems with submodeling or "structural zoom" techniques which can be interpreted as not iterating in the Global/Local coupling.

The Reference and Global meshes contain about 500000 degrees of freedom (dof), the zone of interest contains 80000 dof, and the interface is about 6400 dof large. Only one increment of load (pressure applied to one face) is considered. 


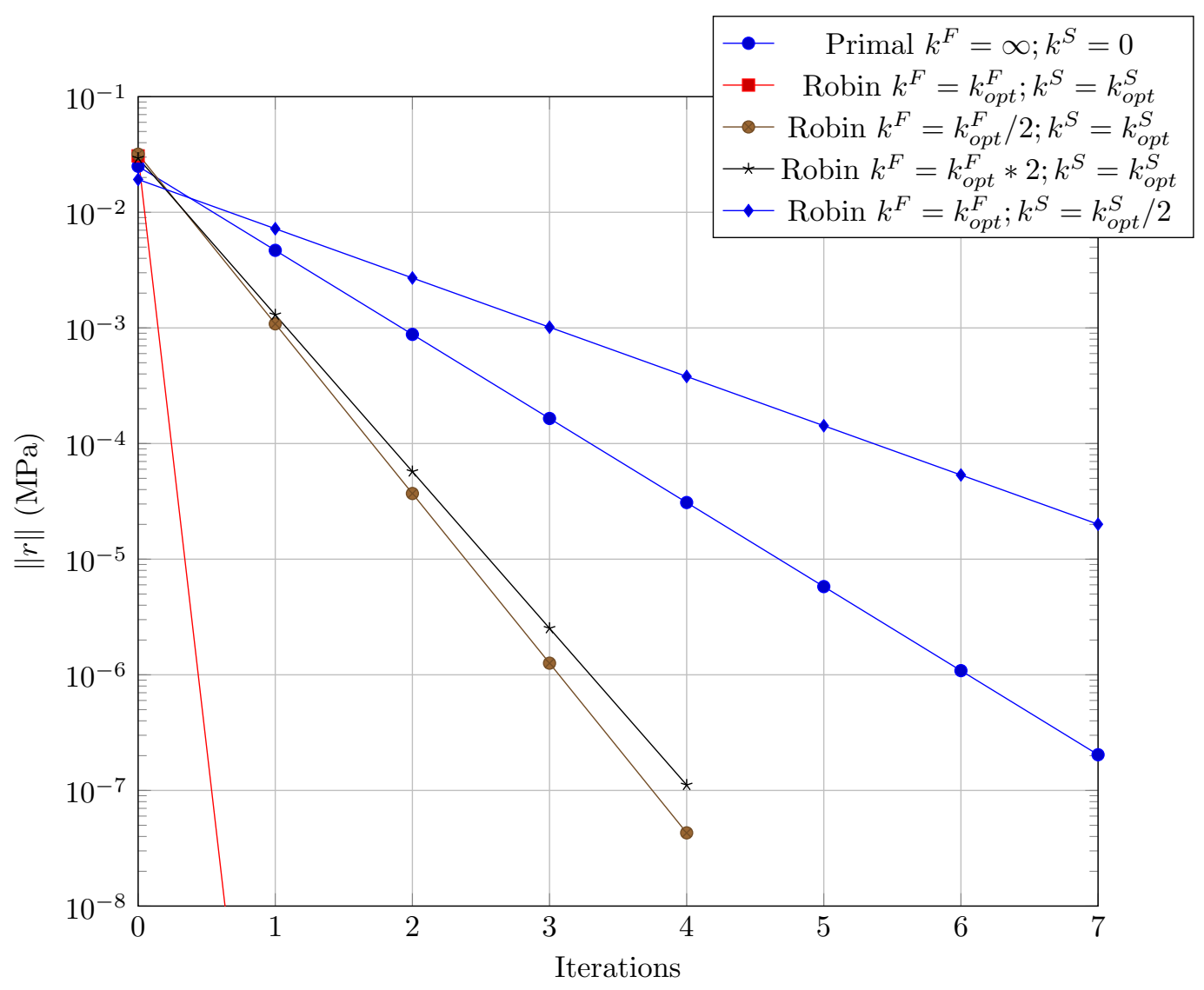

Figure 6: Comparison between various Robin techniques

The computation is managed by a python script which drives Abaqus software. The implementation is non-intrusive in the sense that the models are barely modified, only the extra interface load must be added to the global model. The two-scale Robin approach of Gendre et al. (2011) consists in extending the Fine model with 3 layers of (elastic) elements to approximate the surrounding stiffness; the long-scale effects are taken into account by a projector based on the response of the global structure to SaintVenant loads on the interface. When needed, Sherman-Morrison is applied to take into account low-rank alteration to the stiffness matrix.

The problem was small enough in order to compute the Reference solution. Figure 9 presents the convergence of the method, measured by the error in terms of maximum accumulated plastic strain compared to the reference. The basic iteration takes 10 iterations to lower the error by 2 order of magnitudes. The accelerated version (SR1 quasi-Newton) needs 7 iterations to lower the error by 5 orders of magnitudes. Finally, the Robin version with acceleration only needs 4 iterations for a 6 -order of magnitude decrease.

Note that a plateau can be observed around a relative error of $10^{-6}$. This is very common when using industrial software: some truncation was applied by Abaqus on strain and stress which makes it impossible to achieve better precision. Often, the estimation of the residual (nodal forces) does not suffer such limitation, so that the residual can be decreased up to machine zero whereas the actually attainable mechanical precision was reached much earlier.

We end up the illustration by important mechanical considerations. Figures 10 and 11 present a comparison between the solution obtained by the classical submodeling approach and the Global/Local coupling. In the presented case, the classical submodeling, widely used by industrialists, provides a good estimation of the stress in the zone of interest. But it is really inaccurate in terms of plastic equivalent strain which, for the record, is one of the mechanical quantity used to estimate the lifespan of the structure under cyclic loading. Global/Local coupling is thus a powerful tool to achieve higher precision in the computation without impeding the industrialists' design chain, since usual tools can be employed without profound alteration.

Note that the Global/Local coupling can also provide a simple framework in order to introduce dedicated software for localized phenomena, see e.g. Guguin et al. (2016) for the use of a research code 


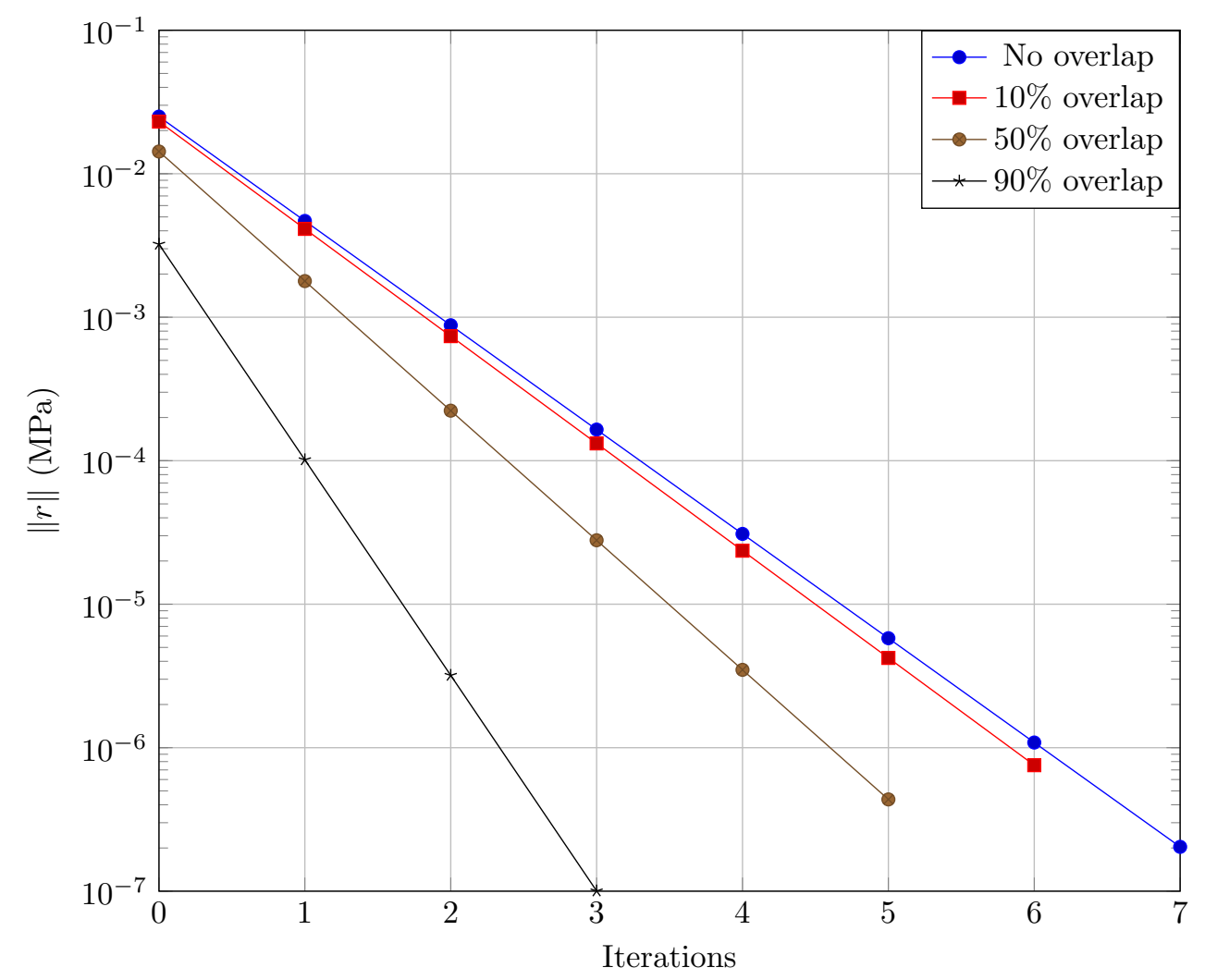

Figure 7: Comparison between different overlap sizes (basic iteration).

specialized in friction contact simulation to precisely model bolts in composite a plate assembly.

\section{Conclusion}

So far, most of what has been done regarding the global/local non-invasive coupling technique concerns the development of the method within legacy codes. We hope that the very simple case which was analyzed in this paper will be useful for anyone, and possibly $\mathrm{PhD}$ students, to get familiar with the method and to further develop it. We think in particular to its initial motivation: to make it possible realistic simulation of complex industrial problems including all their physical and technological complexity. The proposed method should allow, by an easy and fast coupling, to merge research software, with their enhanced physical capabilities, with industrial ones with their geometrical and technological capabilities.

\section{References}

Olivier Allix. A composite damage meso-model for impact problems. Composites Science and Technology, 61:2193-2205, 2001.

Omar Bettinotti, Olivier Allix, and Benoît Malherbe. A coupling strategy for adaptive local refinement in space and time with a fixed global model in explicit dynamics. Computational Mechanics, 53(4): $561-574,2014$.

Omar Bettinotti, Olivier Allix, Umberto Perego, Victor Oancea, and Benoit Malherbe. Simulation of delamination under impact using a global local method in explicit dynamics. Finite Elements in Analysis and Design, 125(8):1-13, 2017.

Maxime Blanchard, Olivier Allix, Pierre Gosselet, and Geoffrey Desmeure. Space/time global/local noninvasive coupling strategy: Application to viscoplastic structures. Finite Elements in Analysis and Design, 156:1-12, 2019. doi: 10.1016/j.finel.2019.01.003. 


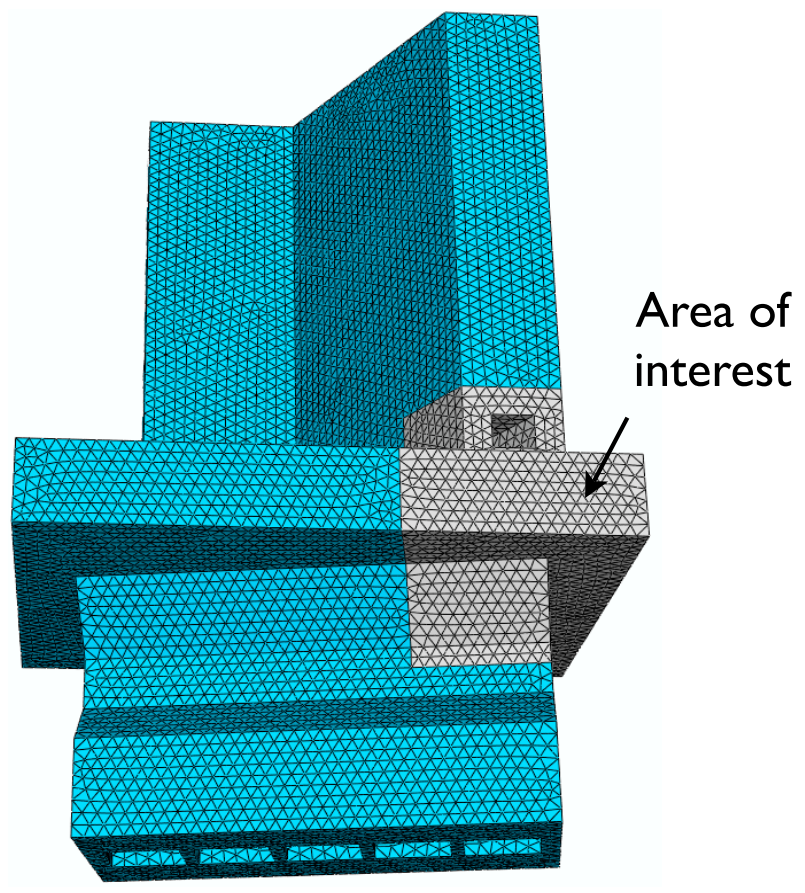

Figure 8: Sweded turbine blade

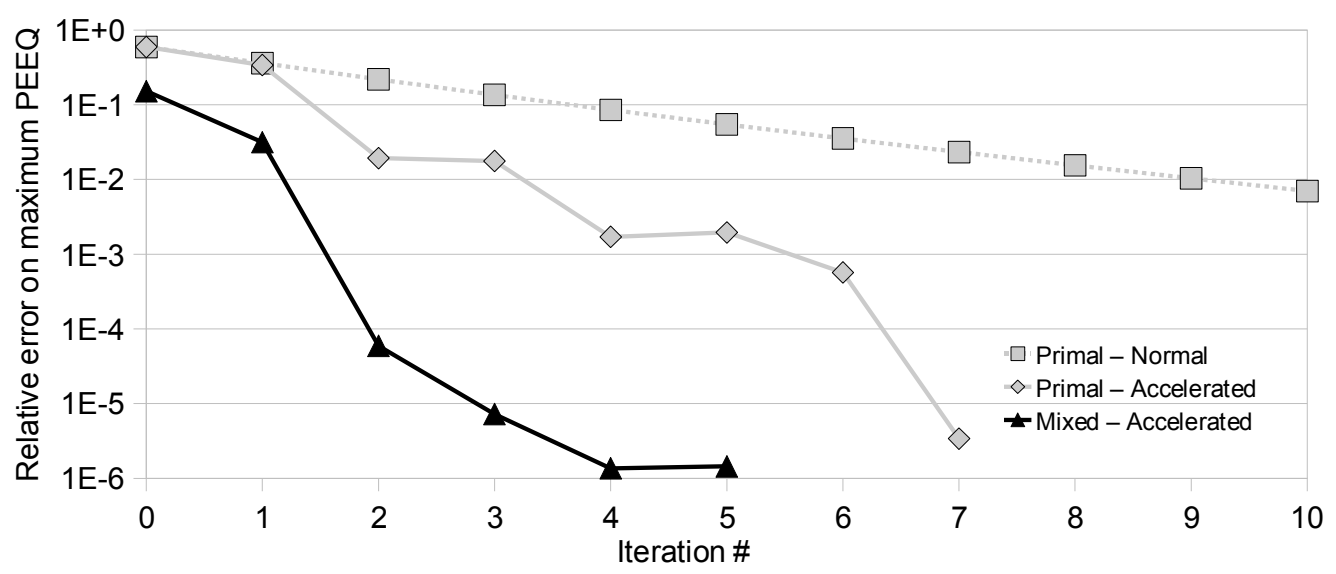

Figure 9: Convergence on the 3D test case - error in accumulated plastic strain

N. G. Cormier, B. S. Smallwood, G. B. Sinclair, and G. Meda. Aggressive submodelling of stress concentrations. International Journal for Numerical Methods in Engineering, 46(6):889-909, 1999. ISSN 1097-0207.

Hachmi Ben Dhia. Problèmes mécaniques multi-échelles: la méthode Arlequin. Comptes Rendus de l'Académie des Sciences - Series IIB - Mechanics-Physics-Astronomy, 326(12):899 - 904, 1998.

Mickaël Duval, Jean-Charles Passieux, Michel Salaün, and Stéphane Guinard. Non-intrusive coupling: recent advances and scalable nonlinear domain decomposition. Archives of Computational Methods in Engineering, 23(1):17-38, 2016.

Mickael Duval, Alexei Lozinski, Jean-Charles Passieux, and Michel Salaün. Residual error based adaptive mesh refinement with the non-intrusive patch algorithm. Computer Methods in Applied Mechanics and Engineering, 329:118-143, 2018.

Treavis B. Fillmore and C. Armando Duarte. A hierarchical non-intrusive algorithm for the generalized finite element method. Advanced Modeling and Simulation in Engineering Sciences, 5(2), 2018.

Lionel Gendre, Olivier Allix, Pierre Gosselet, and François Comte. Non-intrusive and exact global/local techniques for structural problems with local plasticity. Computational Mechanics, 44(2):233-245, 2009. 

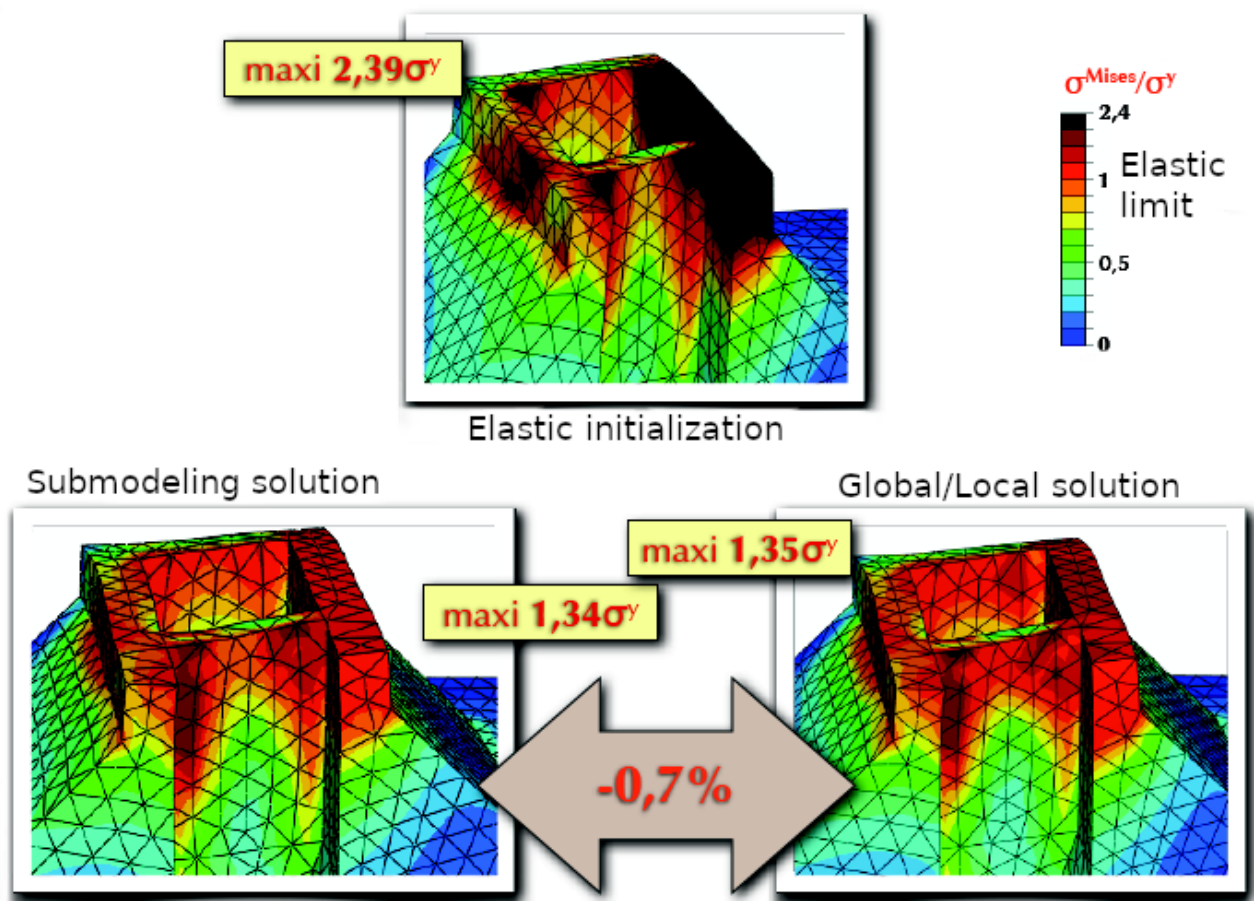

Figure 10: Comparison between submodeling of Global/Local coupling: von Mises stress

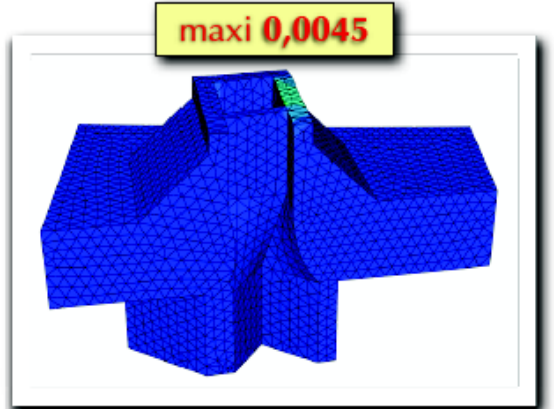

Submodeling solution

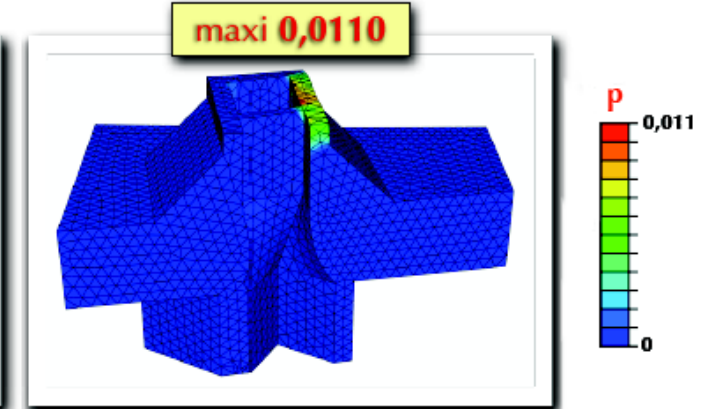

Global/Local solution

Figure 11: Comparison between submodeling of Global/Local coupling: accumulated plastic strain

Lionel Gendre, Olivier Allix, and Pierre Gosselet. A two-scale approximation of the Schur complement and its use for non-intrusive coupling. International Journal for Numerical Methods in Engineering, 87(9):889-905, 2011.

T. Gerasimov, N. Noii, and O. et al. Allix. A non-intrusive global/local approach applied to phase-field modeling of brittle fracture. Advanced modeling and simulation in engineering sciences, 5:1, 2018.

Pierre Gosselet, Maxime Blanchard, and Olivier Allix. Non-invasive global-local coupling as a schwarz domain decomposition method: acceleration and generalization. Advanced Modeling and Simulation in Engineering Sciences, 5(4), 2018a. hal-01613966v1.

Pierre Gosselet, Maxime Blanchard, Olivier Allix, and Guillaume Guguin. Non-invasive global-local coupling as a Schwarz domain decomposition method: acceleration and generalization. Advanced Modeling and Simulation in Engineering Sciences, 5(4), 2018b. doi: 10.1186/s40323-018-0097-4.

Guillaume Guguin, Olivier Allix, Pierre Gosselet, and Stéphane Guinard. Nonintrusive coupling of 3d and $2 \mathrm{~d}$ laminated composite models based on finite element 3d recovery. International Journal for Numerical Methods in Engineering, 98(5):324-343, 2014.

Guillaume Guguin, Olivier Allix, Pierre Gosselet, and Stéphane Guinard. On the computation of plate 
assemblies using realistic 3d joint model: a non-intrusive approach. Adv. Model. and Simul. in Eng. Sci., 3, 2016.

Stéphane Guinard, Robin Bouclier, Mateus Toniolli, and Jean-Charles Passieux. Multiscale analysis of complex aeronautical structures using robust non-intrusive coupling. Advanced Modeling and Simulation in Engineering Sciences, 5(1), 2018.

M. Holl, S. Loehnert, and P Wriggers. An adaptive multiscale method for crack propagation and crack coalescence. International Journal for Numerical Methods in Engineering, 93(1):23-51, 2013.

S Hühne, J Reinoso, Eelco Jansen, and et al. A two-way loose coupling procedure for investigating the buckling and damage behaviour of stiffened composite panels. Composite Structures, 136:513-525, 2016.

Y.J. Liu, Q. Sun, and X.L. Fan. A non-intrusive global/local algorithm with non-matching interface: Derivation and numerical validation. Comput. Methods Appl. Mech. Engrg., 277:81-103, 2014.

J.T. Oden, T. Belytschko, J. Fish, T.J.R. Hughes, C. Johnson, D. Keyes, A. Laub, L. Petzold, D. Srolovitz, and S.Yip. Simulation-based engineering science: Revolutionizing engineering science through simulation. NSF Blue Ribbon Panel on SBES, 2006.

Jean-Charles Passieux, Julien Réthoré, Anthony Gravouil, and Marie-Christine Baietto. Local/global non-intrusive crack propagation simulation using a multigrid x-fem solver. Computational Mechanics, 52(6):1381-1393, 2013

J. Plews, C. A. Duarte, and T. Eason. An improved non-intrusive global-local approach for sharp thermal gradients in a standard fea platform. International Journal for Numerical Methods in Engineering, 91 (4):361-397, 2012.

I. Temizer and P. Wriggers. An adaptive multiscale resolution strategy for the finite deformation analysis of microheterogeneous structures. Computer Methods in Applied Mechanics and Engineering, 200(3740):2639 - 2661, 2011. ISSN 0045-7825. Special Issue on Modeling Error Estimation and Adaptive Modeling. 